Received: 2016.11 .06

Accepted: 2017.01 .20

Published: 2017.11.17

\section{Diagnosis of Acute Appendicitis Using Clinical Alvarado Scoring System and Computed Tomography (CT) Criteria in Patients Attending Gujarat Adani Institute of Medical Science - A Retrospective Study}

\author{
Krishnkant Vaghela ${ }^{\text {ABCDEFE }}$, Bhaven Shah ${ }^{\text {CEF }}$ \\ Department of Radiology, Gujarat Adani Institute of Medical Science, Bhuj, Kutch, Gujarat
}

Author's address: Krishnkant Vaghela, Department of Radiology, Gujarat Adani Institute of Medical Science, Bhuj, Kutch, Gujarat, e-mail: drpiyushpujara@gmail.com
D Data

E Manuscript Preparation

F Literature Search

G Funds Collection

\section{Summary}

Background: $\quad$ The aim was to evaluate the clinical Alvarado scoring system and computed tomography (CT) criteria for the diagnosis of acute appendicitis.

Material/Methods: $\quad$ The study was carried out retrospectively in patients who were referred to the Institute between March 2014 and January 2015. One hundred seventeen patients with acute abdominal pain who underwent abdominal CT were enrolled in this retrospective study. Patient demographics, clinical Alvarado scoring, CT images, and pathologic results of the patients were evaluated.

Results: Thirty nine of the 53 patients who were operated on had pathologically proven acute appendicitis. CT criteria of appendiceal diameter, presence of periappendiceal inflammation, fluid, appendicoliths, and white blood cell count (WBC) were significantly correlated with the inflammation of the appendix. The optimal cut-off value of the appendiceal diameter was $6.5 \mathrm{~mm}$. The correlation between appendiceal diameter and WBC was $80 \%(P=0.01<0.05)$. The correlation between appendiceal diameter and Alvarado score was 78.7\% $(P=0.01<0.05)$.

Conclusions: $\quad$ Presence of appendiceal diameter above $6.5 \mathrm{~mm}$ on CT, periappendiceal inflammation, fluid, and appendicoliths should prompt the diagnosis of acute appendicitis. Since patients with acute appendicitis may not always show the typical signs and symptoms, CT is a helpful imaging modality for patients with relatively low Alvarado scores and leukocytosis, when physical examination is confusing.

MeSH Keywords:

Appendix • Background Radiation • Dental Technicians

PDF file:
http://www.polradiol.com/abstract/index/idArt/902246

\section{Background}

Appendicitis is an inflammation of the appendix, a 3.5inch long tube of tissue that extends from the large intestine. There is no certainty with respect to the function of the appendix [1]. Appendicitis is a medical emergency that requires prompt surgery to remove the appendix. If left untreated, an inflamed appendix will eventually burst or perforate, thereby spilling infectious material into the abdominal cavity. This can lead to peritonitis, a serious inflammation of the abdominal cavity's lining (the peritoneum) that can be fatal, unless it is treated quickly with strong antibiotics [2].
Sometimes, a pus-filled abscess (infection that is walled off from the rest of the body) forms outside the inflamed appendix. Then, scar tissue "walls off" the appendix from the rest of the abdomen, preventing infection from spreading. An abscessed appendix is a less urgent situation, but unfortunately, it cannot be identified without surgery. For this reason, all cases of appendicitis are treated as emergencies that require surgery [3].

Appendicitis occurs when the appendix becomes blocked, often by stool, a foreign body, or cancer. Blockage may also occur from infection, since the appendix swells in response to any infection in the body [4]. 
Acute appendicitis is the most common cause of acute surgical abdomen, with an estimated life-long risk of $8.6 \%$ in men and $6.7 \%$ in women [5]. It is often regarded as a disease of the young, with a peak incidence in the second and third decades of life [6]. Appendectomy is generally accepted as the first-line treatment for non-complicated acute appendicitis. Reports have shown that preoperative radiographic evaluation has helped to decrease negative appendectomy rates from $20 \%$ to as low as 5\% [7]. Computed tomography (CT) has been frequently used as an imaging modality in the evaluation of acute appendicitis and has improved the diagnostic ability, thereby leading to a significant reduction in the number of negative appendectomies [8]. With a reported sensitivity of up to $96.5 \%$ and specificity of about $98 \%$, CT plays a major role in the clinical decision-making process in acute appendicitis and is considered as the first-line imaging modality in the diagnostic work-up for suspected acute appendicitis [9-11].

Diagnosing appendicitis can be difficult. Symptoms of appendicitis are frequently vague or extremely similar to other ailments, including gallbladder problems, bladder or urinary tract infection, Crohn's disease, gastritis, intestinal infection, and ovary problems. The following tests are usually used to make the diagnosis: 1) abdominal exam to detect inflammation, 2) urine test to rule out a urinary tract infection, 3) rectal exam, 4) blood tests to look for signs of infection, 5) CT and/or ultrasound.

In 1986, Alvarado presented a clinical scoring system, based on eight predictive clinical factors, to improve the accuracy of diagnosing acute appendicitis. This scoring system produces a maximum total score of 10 points and includes clinical symptoms, signs, and laboratory findings. Right lower quadrant pain and leukocytosis contribute 2 points each, while the remaining factors contribute 1 point each [12].

The aim of the study was to analyze CT and clinical Alvarado scoring system criteria and to determine an optimal cut-off value of appendiceal diameter for the diagnosis of acute appendicitis.

\section{Material and Methods}

The study was carried out retrospectively in patients who were referred to our Institute between March 2014 and January 2015. Following the approval of the institutional review board, the research was carried out. The following data were analyzed: patient demographics, Alvarado clinical assessment scoring, and radiologic and pathologic results of the patients who had undergone abdominal CT for acute abdomen in our hospital.

The Alvarado score is a clinical scoring system used in the diagnosis of appendicitis. The score has 6 clinical items and 2 laboratory measurements with a total 10 points. It was introduced in 1986, and although meant for pregnant females, it has been extensively validated in the nonpregnant population. The two most important factors, tenderness in the right lower quadrant and leukocytosis, are assigned two points each, and the six other factors are assigned one point each..
A score of 5 or 6 is compatible with the diagnosis of acute appendicitis. A score of 7 or 8 indicates a probable appendicitis, and a score of 9 or 10 indicates a very probable acute appendicitis.

A total of 117 patients who had abdominal CT for acute abdomen, performed within 24-48 hours after the beginning of acute pain, were analyzed in this retrospective study. CT examinations were performed in patients who were scanned in the supine position from the level of the liver dome to the symphysis pubis. Iodinated contrast medium (100-120 ml) was injected via the antecubital vein at a rate of $3 \mathrm{ml} /$ second, with a delay of 60 seconds between contrast administration and data acquisition. Axial images, 5-mm thick, were obtained. Soft tissue kernel was used and reconstruction increment was $1 \mathrm{~mm}$.

On 5-mm thick axial CT images, we measured the appendiceal diameter and analyzed the presence or absence of inflammation, free fluid, and appendicoliths. Pathological diagnosis was used as the reference standard.

CT evaluation of the appendix was based on four criteria: diameter of the appendix, periappendiceal inflammation, presence of extraluminal fluid collection around the appendix, and appendicoliths [13].

\section{Statistical analysis}

All statistical analyses were performed using SPSS, version 15.0. Patient demographics, laboratory findings, clinical Alvarado scores, and CT interpretations were compared between patients with normal appendix and acute appendicitis. Pearson's chi-squared test was used to analyze the relation to sex; the Mann-Whitney test was used to compare patients with normal appendix and acute appendicitis with respect to age, appendiceal diameter, and white blood cell. The Pearson's chi-square test was used to analyze the association between periappendiceal inflammation, fluid, appendicoliths, and inflammation of the appendix. The Spearman's correlation coefficient was used to evaluate the correlation between appendiceal diameter and WBC.

\section{Results}

Based on a retrospective analysis of patient files, 53 patients underwent appendectomy (male, 37 (69.8\%); female, 16 (30\%); mean age, 43 years; range, 16-72 years). Thirty-nine of them had pathologically proven acute appendicitis (male, 28 (71.8\%); female, 11 (28.2\%); mean age, 41 years; range, 16-72 years). The remaining 14 patients had clinical acute appendicitis as determined by the surgeon, but the histopathology of these patients was normal (Tables 1, 2).

Sixty-four patients with abdominal pain had nonsurgical treatment. According to the follow-up of these patients, 7 patients were diagnosed with nephrolithiasis, 9 with ureterolithiasis, 2 with pyelonephritis, 5 with cholecystitis, 2 with pancreatitis, 8 with subileus, 14 with diverticulitis, 2 with ulcerative colitis, 3 with Crohn's disease, 5 with mesenteric lymphadenitis, 1 with epiploic appendicitis, 2 with ovarian cyst, 1 with pelvic inflammatory disease, and 3 with familial Mediterranean fever. 
Table 1. Demographic characteristics of patients.

\begin{tabular}{cc}
\hline Sex & Number \\
\hline Male & 37 \\
\hline Female & 16 \\
\hline Total & 53 \\
\hline
\end{tabular}

Table 2. Diagnosis in patients.

\begin{tabular}{lc}
\hline \multicolumn{1}{c}{ Diagnosis } & Number \\
\hline Pathologically diagnosed appendicitis & 39 \\
\hline Clinically acute appendicitis & 13 \\
\hline Total & 53 \\
\hline
\end{tabular}

Table 3. Correlation between symptoms and CT evaluation.

\begin{tabular}{lccc}
\multicolumn{1}{c}{ Symptoms } & \multicolumn{2}{c}{ Percentage } & P value \\
\hline Mild to moderate periappendiceal inflammation & 10 & $(12.8 \%)$ & \\
\hline Severe periappendiceal & 3 & $(3.8 \%)$ & 0.03 \\
\hline Normal appendix Periappendiceal fluid & 9 & $(11.5 \%)$ & \\
\hline Acute appendix Periappendiceal fluid & 15 & $(38.5 \%)$ & \\
\hline
\end{tabular}

$P$ value $<0.05$, statistically significant.

When the patients with pathologically proven acute appendicitis were analyzed, sex was not related to the inflammation of the appendix. The age of patients was also not related to the inflammation of the appendix. $(p>0.05)$

Appendiceal diameter and white blood count (WBC) were correlated with the inflammation of the appendix $(\mathrm{p} \leq 0.05)$. There was a correlation between the presence of periappendiceal inflammation on CT and the presence of fluid, appendicoliths, and inflammation of the appendix $(\mathrm{p} \leq 0.05))$. In the patients with normal appendix, mild to moderate periappendiceal inflammation was noted in 10 patients $(12.8 \%)$, and severe periappendiceal inflammation was present in 3 patients (3.8\%). Nine patients (11.5\%) with normal appendix presented with periappendiceal fluid; on the other hand, 15 patients (38.5\%) with acute appendicitis had periappendiceal fluid. The optimal cut-off value of appendiceal diameter was found to be $6.5 \mathrm{~mm}$, with a very high prediction rate. The correlation between appendiceal diameter and WBC was $80 \%(\mathrm{p} \leq 0.05)$. The correlation between appendiceal diameter and the Alvarado score was 78.7\% $(\mathrm{p} \leq 0.05)$ (Table 3).

\section{Discussion}

This study analyzed CT criteria and clinical Alvarado scoring system in order to find out the optimal cut-off value of appendiceal diameter for the diagnosis of acute appendicitis. CT diagnosis of acute appendicitis can be based on four criteria which include appendiceal diameter, presence of appendicoliths, periappendiceal inflammation, and free fluid [14]. It is crucial to determine the maximum diameter of appendix with CT for accurate diagnosis of acute appendicitis and to eliminate other etiologies of acute abdominal pain. The inflamed appendix is distended, with a diameter between 6 and $40 \mathrm{~mm}$ and a wall thickness of 1-3 $\mathrm{mm}$ [15]. The wall is usually asymmetrically thickened and enhances after administration of intravenous contrast [16]. In this research, the optimal cut-off value of appendiceal diameter was found to be $6.5 \mathrm{~mm}$, and there was a significant correlation between appendiceal diameter and the Alvarado score.

Appendicoliths detected on CT are reported to be associated with severe appendicitis, appendiceal perforation, recurrent appendicitis after conservative therapy, or failure of antibiotic therapy [17]. Ishiyama et al. showed a significant relationship between the presence of appendicoliths and the severity of acute appendicitis in a retrospective study with a total of 254 patients who had pathologically proven acute appendicitis [17]. The authors suggested that it is probable that the root of the appendix may be easily obstructed by an appendicolith, as the root of the appendix has a narrower lumen in comparison to the rest of the appendix. They further asserted that an appendicolith can lead to severe disease, especially when it is a larger one or is located at the root of the appendix.

There are some specific CT findings that should raise surgeons' suspicion of perforation, which can be a complication of appendicitis. Other complications include abscesses, phlegmon, extraluminal air, extraluminal appendicolith, and focal defect in the enhanced wall of the appendix.

On CT, ascending retrocecal appendicitis has been reported to be associated with a high incidence of retroperitoneal inflammatory changes and appendiceal perforation. Periappendiceal inflammatory changes are most commonly observed in the retrocolic space, followed by paracolic gutter, pararenal space, mesentery, perirenal space, and subhepatic space. Perforation of the appendix with the formation of an abscess is present in approximately half of the cases [18].

The utility of Alvarado scoring system has been widely researched. In a review of 233 patients with right lower quadrant pain, Pouget-Baudry et al. established that Alvarado scoring was very useful. The authors found out that a score of 6 correlated well with the presence of appendicitis, and score 4 correlated well with the absence of appendicitis. They suggested that observation or 
complementary tests should be used only in the case of a score between 4 and 6 [19]. McKay and Shepherd recommended surgical consultation if clinical presentation suggested acute appendicitis by an Alvarado score of 7 or higher. They reported that computed tomography was not indicated for diagnosing acute appendicitis in patients with Alvarado scores of 3 or lower [20]

Wang et al. researched the use of CT in patients with suspected acute appendicitis who had relatively low Alvarado scores [21]. Sixty patients with suspected acute appendicitis and an Alvarado score between 4 and 7 points were considered in a prospective study. Clinical and laboratory differences were compared between patients with histologically proven acute appendicitis and patients without acute appendicitis. The authors evaluated whether the use of CT could be decreased in patients who were less likely to have acute appendicitis. They concluded that CT is necessary for patients with relatively low Alvarado scores, when leukocytosis is noted.

Nelson et al. carried out a retrospective study to examine the relevance of clinical assessment in diagnosing appendicitis in the era of routine use of CT in a total of 664 patients. In cases of high clinical suspicion, the surgeon's clinical assessment was reliable, whereas if the surgeon's initial impression was low for acute appendicitis, 87\% of these patients had confirmed appendicitis on final pathology. The authors concluded that although physical examination remains crucial, CT has become the primary modality dictating care of patients with presumed appendicitis [22].

Elderly patients may account for nearly $10 \%$ of cases referred for CT for suspected appendicitis [23]. The classic presentation of appendicitis involving the triad of fever, leukocytosis, and right lower quadrant pain is present in only $10-26 \%$ of patients over 60 years of age. Treating elderly patients may pose a challenge, since surgery is the treatment modality in the majority of cases of acute appendicitis. Given that elderly patients are more prone to have relevant comorbidities, the elderly are at an increased risk for complications related to both delayed diagnosis of appendicitis and unnecessary appendectomy. The overall mortality rate for elderly patients with appendicitis has been reported to be about 15\% [24]. An accurate diagnostic test for acute appendicitis is therefore very crucial in elderly patients with suspected appendicitis.

An accurate diagnosis of acute abdomen is important in distinguishing surgical conditions like acute appendicitis from nonsurgical conditions that may have a similar presentation. Various pathologies might mimic appendicitis on CT imaging. These include right-sided diverticulitis, cecal carcinoma, Crohn's colitis, mesenteric inflammation, complicated ovarian cysts, endometriosis, ectopic pregnancy, local lymphadenopathy, and fibrofatty proliferation [25]. Perforated duodenal ulcer, superior mesenteric venous thrombosis, small bowel ischemia, and abdominal wall hernia are conditions which present with right lower abdominal pain and are treated surgically [26]. A surgeon's clinical evaluation alone can reliably diagnose acute appendicitis in highly suspicious cases of appendicitis without the help of CT. Nevertheless the surgeon's assessment may miss patients who meet only few diagnostic criteria. In these patients, CT becomes an effective and useful adjunct in the workup of acute appendicitis. Without the use of CT, the rate of negative appendectomy has been reported as high as $17-36 \%$ [27]. The negative appendectomy rate in our study was $26.41 \%$, which was relatively high and needs to be lowered with further research and quality monitoring.

Radiologic diagnosis of acute appendicitis can be missed, especially when patients have equivocal CT findings [28]. Appendicitis is present in up to $30 \%$ of patients with equivocal CT findings. As a result, in spite of the progress in CT techniques, negative appendectomy and delayed diagnosis may still occur.

A recent meta-analysis of CT use in the evaluation of suspected acute appendicitis suggested that routine CT in all patients presenting with suspected appendicitis could reduce the rate of unnecessary surgery without increasing morbidity [29]. In the diagnosis of suspected acute appendicitis, CT has been reported to decrease the incidence of negative appendectomy [29].

The role of ultrasonography should also be emphasized in the diagnosis of acute appendicitis, since it is a widely available and affordable modality which does not utilize ionizing radiation. It has been reported to have a sensitivity between $55-98 \%$ and specificity of $78-100 \%$. The limitations of this technique are user dependency and the difficulty in obtaining good image quality in some patients [30].

\section{Conclusions}

CT is an accurate imaging modality for the diagnosis of acute appendicitis. Presence of CT criteria of appendiceal diameter above $6.5 \mathrm{~mm}$, periappendiceal inflammation, fluid, and appendicoliths should prompt the diagnosis of acute appendicitis. Even though the optimal use of CT in evaluating patients with suspected appendicitis is not clear, it is necessary in patients with relatively low Alvarado scores and leukocytosis and also when physical examination is confusing. The sample size in the present study was very small and more emphasis should be put on studies with larger sample sizes, on the basis of which, more accurate results can be obtained.

\section{References:}

1. Boyer RC: Abdominal pain. 1937

2. Quick CR, Reed JB, Harper SJ et al: Essential surgery: Problems, diagnosis and management: With STUDENT CONSULT Online Access: Elsevier Health Sciences, 2013
3. Santry HP, Pringle PL, Collins CE, Kiefe CI: A qualitative analysis of acute care surgery in the United States: it's more than just "a competent surgeon with a sharp knife and a willing attitude". Surgery, 2014; 155: 809-25

4. Hunt VC: Torsion of appendices epiploicae. Ann Surg, 1919; 69: 31 
5. Addiss DG, Shaffer N, Fowler BS, Tauxe RV: The epidemiology of appendicitis and appendectomy in the United States. Am J Epidemiol, 1990; 132: 910-25

6. Rybkin AV, Thoeni RF: Current concepts in imaging of appendicitis. Radiol Clin North Am, 2007; 45: 411-22

7. Florence M, Flum DR, Jurkovich GJ et al: Negative appendectomy and imaging accuracy in the Washington state surgical care and outcomes assessment program. Ann Surg, 2008; 248: 557-63

8. Lai V, Chan WC, Lau HY et al: Diagnostic power of various computed tomography signs in diagnosing acute appendicitis. Clin Imaging, 2012; 36: 29-34

9. Raman SS, Lu DS, Kadell BM et al: Accuracy of nonfocused helical CT for the diagnosis of acute appendicitis: A 5-year review. Am J Roentgenol, 2002; 178: 1319-25

10. Tsze DS, Asnis LM, Merchant RC et al: Increasing computed tomography use for patients with appendicitis and discrepancies in pain management between adults and children: An analysis of the NHAMCS. Ann Emerg Med, 2012; 59: 395-403

11. Gunes Tatar I, Yilmaz KB, Sahin A et al: Evaluation of clinical Alvarado scoring system and CT criteria in the diagnosis of acute appendicitis. Radiol Res Pract, 2016; 2016: 9739385

12. Alvarado A: A practical score for the early diagnosis of acute appendicitis. Ann Emerg Med, 1986; 15: 557-64

13. Pinto Leite N, Pereira JM, Cunha R et al: CT evaluation of appendicitis and its complications: imaging techniques and key diagnostic findings. Am J Roentgenol, 2005; 185: 406-17

14. Bursali A, Araç M, Öner AY et al: Evaluation of the normal appendix at low-dose non-enhanced spiral CT. Diagn Interv Radiol, 2005; 11(1): 45-50

15. Rao PM, Rhea JT, Novelline RA et al: Helical CT combined with contrast material administered only through the colon for imaging of suspected appendicitis. Am J Roentgenol, 1997; 169: 1275-80

16. Yeung K-W, Chang M-S, Hsiao C-P: Evaluation of perforated and nonperforated appendicitis with CT. Clin Imaging, 2004; 28: 422-27

17. Shindoh J, Niwa H, Kawai K et al: Predictive factors for negative outcomes in initial non-operative management of suspected appendicitis. J Gastrointest Surg, 2010; 14: 309-14

18. Kim S, Lim HK, Lee JY et al: Ascending retrocecal appendicitis: Clinical and computed tomographic findings. J Comput Assist Tomogr, 2006; 30: 772-76
19. Pouget-Baudry Y, Mucci S, Eyssartier E et al: The use of the Alvarado score in the management of right lower quadrant abdominal pain in the adult. J Visc Surg, 2010; 147: e40-4

20. McKay R, Shepherd J: The use of the clinical scoring system by Alvarado in the decision to perform computed tomography for acute appendicitis in the ED. Am J Emerg Med, 2007; 25: 489-93

21. Wang S-Y, Fang J-F, Liao C-H et al: Prospective study of computed tomography in patients with suspected acute appendicitis and low Alvarado score. Am J Emerg Med, 2012; 30: 1597-601

22. Nelson DW, Causey MW, Porta CR et al: Examining the relevance of the physician's clinical assessment and the reliance on computed tomography in diagnosing acute appendicitis. Am J Surg, 2013; 205: 452-56

23. Pickhardt PJ, Lawrence EM, Pooler BD, Bruce RJ: Diagnostic performance of multidetector computed tomography for suspected acute appendicitis. Ann Intern Med, 2011; 154: 789-96

24. Storm-Dickerson TL, Horattas MC: What have we learned over the past 20 years about appendicitis in the elderly? Am J Surg, 2003; 185: 198-201

25. Mirilas P, Skandalakis JE: Urogenital diaphragm: An erroneous concept casting its shadow over the sphincter urethrae and deep perineal space. J Am Coll Surg, 2004; 198: 279-90

26. Duran JC, Beidle TR, Perret R et al: CT Imaging of acute right lower quadrant disease. Am J Roentgenol, 1997; 168: 411-16

27. Mariadason J, Wang W, Wallack M et al: Negative appendicectomy rate as a quality metric in the management of appendicitis: Impact of computed tomography, Alvarado score and the definition of negative appendicectomy. Ann R Coll Surg Engl, 2012; 94: 395-401

28. Levine CD, Aizenstein O, Lehavi O, Blachar A: Why we miss the diagnosis of appendicitis on abdominal CT: Evaluation of imaging features of appendicitis incorrectly diagnosed on CT. Am J Roentgenol, 2005; 184: 855-59

29. Brandt M-M, Wahl WL: Liberal use of CT scanning helps to diagnose appendicitis in adults. Am Surg, 2003; 69(9): 727-31; discussion $731-32$

30. Flum DR, Koepsell T: The clinical and economic correlates of misdiagnosed appendicitis: nationwide analysis. Arch Surg 2002; 137: 799-804 